\title{
Coronary artery disease prescribing pattern and risk factor assessment in the patients undergoing angioplasty
}

\author{
Nilay D. Solanki ${ }^{1 *}$, Nisha Patel ${ }^{1}$, Shubha Desai ${ }^{2}$, Varsha Patel ${ }^{3}$
}

\author{
${ }^{1}$ Ramanbhai Patel College of Pharmacy, Charotar University of Science and Technology, Changa, Gujarat, India \\ ${ }^{2}$ Dr. Jivraj Mehta Smarak and Health foundation, Ahmedabad, Gujarat, India \\ ${ }^{3}$ Dr. M. K. Shah Medical College and Research Centre, Ahmedabad, Gujarat, India
}

Received: 01 March 2021

Accepted: 03 April 2021

\section{*Correspondence:}

Dr. Nilay D. Solanki,

Email: nilaysolanki.ph@charusat.ac.in

Copyright: (C) the author(s), publisher and licensee Medip Academy. This is an open-access article distributed under the terms of the Creative Commons Attribution Non-Commercial License, which permits unrestricted non-commercial use, distribution, and reproduction in any medium, provided the original work is properly cited.

\begin{abstract}
Background: Coronary artery disease is caused by an obstruction in vascular supply to the heart. Angioplasty is a frequently used intervention for the management of CAD patients. Supportive and preventive therapies are additionally provided to these patients. Objective of current study was to assess the associated risk factors as well as prescribing trends in CAD patients.

Methods: A prospective study was conducted in 88 patients undergoing coronary angioplasty. Patients were assessed for risk factors of CAD. Medication history of patients was recorded in case record form for analysis of prescribing trend and its rationality. Data were statistically analyzed using prism software.

Results: In present study most of the CAD patients were male $(80.68 \%)$ and mean age of patient was 59.19 years. Majority of patients $(39.77 \%)$ were in age range of $61-70$ years. Most common associated conditions at admission were hypertension and diabetes. In this study, BMI, stress, lack of exercise, hypertension, diabetes, family history of CVD were risk factors of CAD and significant correlation observed with risk factors and coronary artery disease. $(\mathrm{p}<0.05)$ Frequently prescribed drugs were antiplatelet, antibiotics, antihypertensive, antiulcer, antihyperlipidemic, antidiabetic agents. In $19.31 \%$ cases, drug interaction was detected out of which two cases recorded severe. Medical stents coated with everolimus, sirolimus were also prescribed.

Conclusions: It was concluded that history of cardiovascular disease, hypertension, diabetes are major risk factors of CAD. Certain level of irrationality in the prescribing trend was observed. Proper patient counselling and care can help in preventing CAD and reduces health burden.
\end{abstract}

Keywords: Coronary artery disease, Angioplasty, Antiplatelet drugs, Stents, Risk factors

\section{INTRODUCTION}

Coronary artery disease is also called coronary heart disease (CHD) or ischemic heart disease (IHD). IHD is caused by the narrowing of one or more of the main coronary arteries, most commonly atherosclerotic disease plaques, delivering blood to the heart. Coronary artery disease (CAD) in India is becoming significant health issue for the population. An age-standardized study on the global burden of disease showed that almost $24 \%$ of all deaths in India are caused by CAD. Heart and stroke is the most common risk of vascular disease mortality in India $(83 \%){ }^{1-6}$ Hypertension, diabetes mellitus, dyslipidemia, obesity, smoking, sedentary lifestyles, type 2 characteristics, non-modifiable risks, such as age, gender and a history of family disease, are listed as modifiable risk factors for $\mathrm{CAD}^{7} \mathrm{CAD}$ is closely correlated with lifestyles and physiological influences and improvements in risk factor linked to cardiovascular 
morbidity and mortality reduction. Recent rise in diabetes, obesity, and the incidence of IHD is growing at a younger age as well. The current research plans was to determine risk factors and recommend prescribing patterns in CAD at tertiary treatment centre.

\section{METHODS}

A prospective research was conducted on the inpatient department of Dr. Jivraj Mehta Smarak and the health foundation for a period of 7 months from July 2018 to January 2019, after approval by the hospital ethics committee. A total of 88 patients underwent coronary angioplasty of age ranges from 18 and 80 years. Specific clinical notes have been taken of each patient and the case history of the patient has been reviewed for diagnosis and treatment. All relevant results were recorded separately for each patient in case records and a master chart of all relevant patient data was prepared. For the assessment of risk factors and drug patterns, the data analysis was conducted and the rationale was reported and evaluated for the following. Drug interactions have been tested by using a medicinal interaction checker from Medscape. The data were analyzed with Prism software and MS excel. Statistical analyses were conducted, including the combination t-test, chi-square test, $\mathrm{p}<0.05$ was considered as statistically significant.

\section{RESULTS}

In total, eighty-eight patients underwent angioplasty during the seven months of the study and were registered for drug monitoring and risk factors of CAD. The bulk of patients surveyed were in the 61-70-year age range followed by other graphic categories (Figure 1).

Out of a total of 88 patients, $71(80.68 \%)$ were male and $17(19.32 \%)$ were female. The demographic distribution shows that the male patient proportion was higher than the female patient. Hypertension (53\%), diabetes $(39.77 \%)$, hypothyroidism $(9.09 \%)$, Angina $(2.27 \%)$, left ventricular failure $(2.27 \%)$, congestive heart failure $(1.14 \%)$ is presently related to the disorder with CAD (Table 1). In this study the main risk factors of the CAD were BMI $(55.68 \%)$, hypertension $(53.40 \%)$ and diabetes $(39.77 \%)$; stress $(79.54 \%)$, lack of practice $(20.45 \%)$; family history of the CVD $(70.45 \%)$. The risk factors were associated with CAD $(\mathrm{p}<0.05)$ (Table 4).

Table 1: Associated condition in patients with CAD.

\begin{tabular}{|lll|}
\hline Associated conditions $(\mathbf{n = 8 8})$ & $\mathbf{N}$ & $\mathbf{( \% )}$ \\
\hline Hypertension & 47 & 53 \\
\hline Diabetes mellitus & 35 & 39.77 \\
\hline Hypothyroidism & 8 & 9.09 \\
\hline Angina & 2 & 2.27 \\
\hline Left ventricular failure & 2 & 2.27 \\
\hline Congestive cardiac failure & 1 & 1.14 \\
\hline
\end{tabular}

In current study all medical stents were used in coronary angioplasty, coated with everolimus, sirolimus for preventing proliferation around it. Mostly promus element plus $(15.91 \%)$ and ultimaster (14.77\%) stents were used. Various forms of medicines were recommended for treating coronary heart disease in patients suffering from coronary angioplastics such as antiplatelets $(98.86 \%)$, antibiotics $(84.09 \%)$, lipid reducing agents (84.09\%), antihypertensives (75\%), antihistamines (56.82\%), analgesics (55.68\%), antidiabolics (14.77\%), antiemetic (9.09\%). Antiplatelet drugs were widely recommended in all groups. In $99 \%$ of patients with antiplatelet therapy aspirin and other clopidogrel, ticagrelor, prasugrel and tirofiban, 48.86\%, $50 \%, 17.77$ and $4.55 \%$, respectively, were administered. Nitroglycerin (18.18\%), nicorandil (12.5\%) and isosorbiddinitrite $(11.36 \%)$ were most commonly administered antiangina medication. Cefotaxime (50\%) was widely recruited from other antibiotics as cefixime (43.18\%) and cefadroxil (13.64\%). The blockers of $\beta 1$ adrenoceptors $(57.95 \%)$ were widely recommended among all drug groups, followed by angliotensin-II (38.64\%) and ACE (22.73\%) blockers. Lipid-lowering medications such as rosuvastatin and atorvastatin were administered to the sample population of $47.73 \%$ and $43.18 \%$ respectively. Anticoagulant medications like heparin and enoxaparin sodium have been recommended for the sample population of $20.45 \%$ and $10.23 \%$ respectively. Alongside this, a number of miscellaneous antithyroid drugs, anticoagulants, antihistamines and laxatives were prescribed (Table 2).

\section{Table 2: Miscellaneous drugs prescribed in patients} undergoing coronary.

\begin{tabular}{|lll|}
\hline \multirow{3}{*}{ Drug class } & Drugs & $\begin{array}{l}\text { \% of } \\
\text { patients }\end{array}$ \\
\hline \multirow{3}{*}{ Anti-thyroid } & Thyroxine/Levothyroxine & 4.55 \\
\cline { 2 - 3 } & Levothyroxine & 1.14 \\
\cline { 2 - 3 } & Thyroxine & 1.14 \\
\hline \multirow{3}{*}{ Laxative } & Heparin & 20.45 \\
\cline { 2 - 3 } & Enoxaparin sodium & 10.23 \\
\hline \multirow{3}{*}{ Antihistamine } & $\begin{array}{l}\text { Liquid paraffin+sodium } \\
\text { picosulfate }\end{array}$ & 6.82 \\
\cline { 2 - 3 } & Lactulose & 6.82 \\
\cline { 2 - 3 } & Sodium picosulfate & 7.95 \\
\cline { 2 - 3 } & Hydrocortisone & 52.27 \\
\cline { 2 - 3 } & Pheniramine & 17.05 \\
\hline
\end{tabular}

Throughout the medication study a total of 17 drug-drug interactions were observed, of which 8 (47\%), 7 (41\%), and $2(12 \%)$ were moderate, mild, and serious respectively (Figure 3). A total of 88 prescriptions from that $59.83 \%$ of prescribed drugs were not under the 2017 WHO necessary drug list and measures of prescribing practices as per the WHO guidelines were also created. Within 88 prescriptions examined, 858 medicines were prescribed, reflecting an average of around ten medicines 
per prescription. The WHO drug list contains only 346 drugs. Just six drugs with a common name have been prescribed. The average prescription number of antibiotics was 1.05 (Table 3).

Table 3: Prescribing indicators as per WHO guidelines.

\begin{tabular}{|ll|}
\hline Prescription indicators & N \\
\hline Total no. of prescribed drugs & 858 \\
\hline Average no of drugs per prescription & 9.98 \\
\hline Number of antibiotics per prescription & 1.05 \\
\hline $\begin{array}{l}\text { No. of prescribed drugs from essential drug } \\
\text { list }\end{array}$ & 346 \\
\hline No. of drugs prescribed by generic name & 6 \\
\hline
\end{tabular}

Table 4: Risk factors assessment.

\begin{tabular}{|lll|}
\hline Risk factors & $\mathbf{N}(\%)$ & P value \\
\hline BMI & $49(55.68)$ & 0.0004 \\
\hline Lack of exercise & $18(20.45)$ & $<0.0001$ \\
\hline Stress & $70(79.54)$ & 0.0225 \\
\hline Smoking & $18(20.45)$ & 0.0381 \\
\hline Hypertension & $47(53.41)$ & $<0.001$ \\
\hline Diabetes & $35(39.77)$ & $<0.001$ \\
\hline Family history & $62(70.45)$ & 0.0002 \\
\hline
\end{tabular}

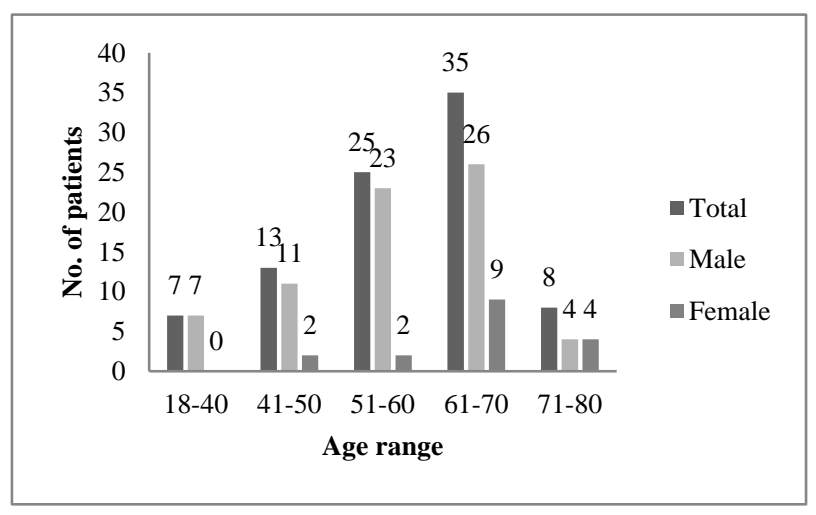

Figure 1: Age and gender wise distribution of patients.

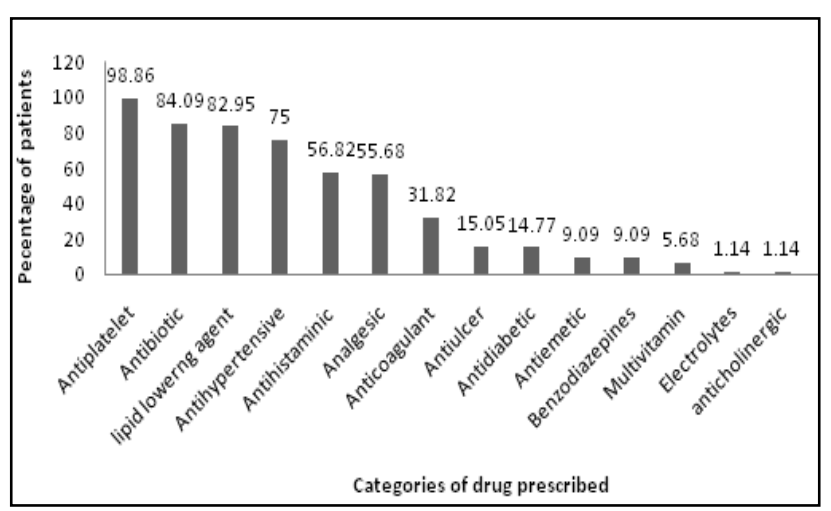

Figure 2: Distribution of prescribed drug categories in patients undergoing coronary angioplasty.

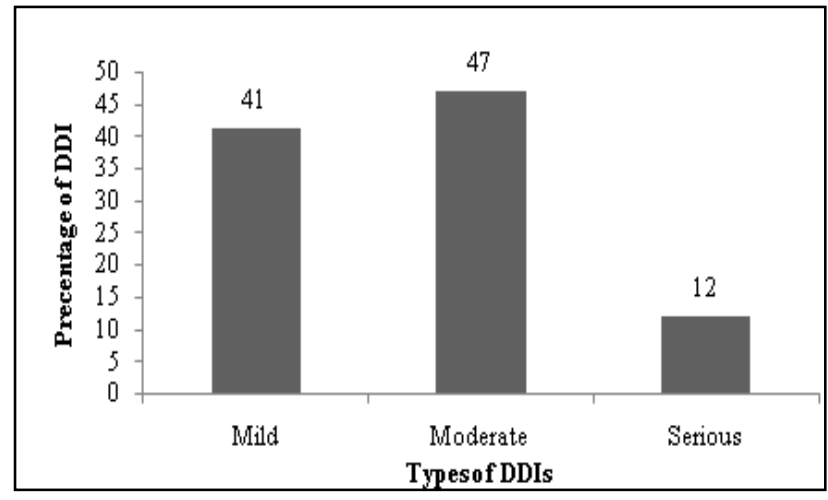

Figure 3: Drug-drug interactions.

\section{DISCUSSION}

Studies of prescribing patterns are useful to identify the problems and provide feedback to prescribers to create awareness about the rational use of drugs. ${ }^{2}$ Therefore, current study analyzed the current prescription patterns of drugs and its rationality in patients undergoing coronary angioplasty and to assess associated risk factors in CAD patients undergoing coronary angioplasty in order to ensure the appropriate use of drugs to decrease the morbidity and mortality of the disease and reduce the economic burden. In the present study, we included 88 patients out of which $71(81 \%)$ were males and $17(19 \%)$ were females with an average age of $59.19 \pm 1.18$ years. Most patients were in the age range of 61-70 years. Similar studies conducted earlier reported higher incidences in males $(68-80 \%)$ than females and the average age of 61.50 years. ${ }^{2,3}$ Thus, the majority of CAD patients' age is above 55 years, and males seem to be more prone to CAD than females. The reason for the low occurrence of CAD in females is normally attributed to the protective effects of estrogen. However, the clinical presentation of CAD in women varies significantly from males, and therefore, many are left undiagnosed. Thus, low occurrence of the disease in females should be taken more cautiously, and more elaborate studies should be conducted to determine the exact cause of such low occurrence. , $^{4-15}$

In the present study for determining family history as a risk factor for CAD, Out of 88 patients, $70.45 \%$ of patients were having a family history of Cardiovascular disease. Smoking was considered as a major risk factor CAD in $20.45 \%$ patients. There was a significant correlation between smoking and coronary artery disease. $(p \leq 0.05)$. Previous studies showed that for smokers under the age of 50 years, the risk of developing CHD is ten times greater than for non-smokers of the same age. The effect of cigarette smoking on coronary risk factors is pervasive. Effect of smoking includes enhancement of platelet function. Platelet activation by cigarette smoking is associated with thrombosis formation, including the onset of myocardial infarction. The adverse effects on platelet function, cessation of smoking should be encouraged. ${ }^{16}$ In current study, the lack of exercise was 
observed as a risk factor CAD. There was a significant correlation between lack of exercise and coronary artery disease $(\mathrm{p} \leq 0.05) .{ }^{17}$ Our study revealed that almost $70 \%$ of patients were having pre-existing cardiovascular disease, diabetes, hypertension, and/or the previous stroke, suggesting that history of CVD must have played a significant role in the development of CAD. Presence of such disease is indicative of pre-existing significant cardiovascular damage and thus has extremely high chances of coronary artery disease. In many epidemiological studies, only parental history is considered for determining family history in CAD patients. However, sibling history also is reported to have a strong association with CAD ${ }^{8,9}$ Another scientific study showed that majority of the hypertensive patients 66 $(57.89 \%)$ have diabetes mellitus (DM) followed by myocardial ischemia $26(22.80 \%)$, thyroid complications $16(14.03 \%)$, asthma $04(3.50 \%)$, chronic obstructive pulmonary disease (COPD) $(0.87 \%)$, and migraine $(0.87)$, which supports our study results. ${ }^{18-21}$

Patients of hypertension, diabetes, hypothyroidism etc. are reported to be at significantly higher risk for CAD. ${ }^{4}$ We observed that $53 \%$ of our study population was already suffering from hypertension. Two studies reported 21 and $25 \%$ of their enrolled CAD patients as hypertensive. ${ }^{5,6}$ The existence of a very high proportion of hypertensive patients in our study also correlates with a stressful lifestyle. Almost $44 \%$ of the subjects were suffering from moderate to severe stress. A similar situation was observed with the co-occurrence of diabetes. Approximately $40 \%$ were diabetics as compared to $15 \%$ reported in previous studies. ${ }^{4,5}$ In the urban city, the reported prevalence of diabetes was quite high $(7.33 \%){ }^{7}$ It was also reported that $23.6 \%$ of diabetic patients develop cardiovascular complications. A significant correlation exists between hypothyroidism and CAD in previous studies. ${ }^{8}$ It was observed that $9 \%$ of current study population was suffering from hypothyroidism. Pre-existence of cardiovascular diseases, mainly angina and CCF was observed in $5.7 \%$ of current study population. All of these complications may have contributed to $\mathrm{CAD}$ requiring angioplasty.

Abnormalities in ECG were observed in more than $90 \%$ of the patients admitted for the angioplasty. Out of this, 30 patients were suffering from myocardial infarction, and the remaining had arrhythmia or heart failure conditions. The reason for this could be secondary complications due to chronic CAD. Stents were commonly placed at the occlusion site to prevent relapses. Large varieties of stents are presently available to meet the needs of all categories of patients. Medicated stents are presently more preferred by the physician. In this study, all medicated stents used were coated with everolimus, and sirolimus used to inhibit organ rejection of transplanted (anti-proliferative). Stents coated with zotarolimus, biolimus drugs are also available. The most commonly used stents were promus element plus $(15.91 \%)$, ultimaster (14.77\%), and synergy (12.5\%).
Stents reduce the number of revascularization procedures and thus provide additional benefit over simple angioplasty. However, the overall mortality within six months is higher in patients vascularized with stents as compared to patients with simple angioplasty. Medicated stents prevent restenosis and thus prevent relapses. ${ }^{9,10}$

The various drugs, used in patients undergoing angioplasty due to myocardial infarction or unstable angina, are prescribed for preventing postoperative infections, for reducing relapses and for treatment of CAD complications. Antiplatelet agents were prescribed in almost all patients for the purpose of preventing relapses. Aspirin alone or in combination with various GPIIB/IIIA inhibitors were used for antiplatelet activity. A short term postoperative antibiotics were prescribed to $85 \%$ of patients for preventing infections. Cephalosporin group of antibiotics were prescribed in all of these cases. Other commonly prescribed drugs included antihypertensives and lipid-lowering agents. Antiulcer drugs were also prescribed in a large number of patients possibly to prevent drug-induced hyperacidity and ulcers. Similar studies on patients suffering from different cardiovascular diseases reported antiplatelet agents as most prescribed, followed by anti-hypertensives. The most prescribed antiplatelet agent was aspirin, followed by clopidogrel. ${ }^{5,11,12}$ In the present study, the commonly prescribed drugs were isosorbide dinitrate among the nitrates, aspirin among the antiplatelet agents, metoprolol among the beta-blockers, amlodipine among the calcium channel blockers, ramipril among the ACE inhibitors, and atorvastatin among the hypolipidaemic. In this study, only the cephalosporin group of antibiotics were prescribed. Management of coronary artery disease involves complex therapeutic treatments. A similar pattern in prescribing of all drugs was observed. Earlier study report, utilization of cephalosporins $(64.56 \%)$, quinolones $(13.92 \%)$, aminoglycosides $(8.86 \%)$, penicillins $(6.96 \%) .{ }^{13}$ In another study for cardiovascular conditions prescribing trends of antiplatelet, Antidyslipidemic agents and Beta blockers were 34\%, $19 \%$ and $14 \%$ respectively, while antianginals, ACE inhibitors and diuretics were prescribed in $11 \%, 8 \%, 5 \%$ respectively, which may be related to our study results. ${ }^{20}$ As a result, drug interactions are a major concern in these patients. Drug-drug interaction was analysed using Medscape drug interaction checker. In this study, out of 88 cases, 17 drug interactions were noted. In past studies frequently prescribed antibiotic group was beta-lactam drug in the accidental cases in which antibiotics were prescribed in a higher ratio. And no serious drug interaction was found, while all the possible drug interactions occur through the pharmacokinetic mechanism. ${ }^{19}$ In present study the severity of drug interaction was classified as mild, moderate and serious. Mild drug interactions were $41 \%$, moderate drug interaction was $47 \%$, and serious interactions were $12 \%$. The serious drug interaction included a co-prescription of aspirin+ramipril and esomeprazole+clopidogrel. Aspirin and ramipril combination can result in a reduction in 
renal function, which may become life-threatening. Similarly, a combination of esomeprazole and clopidogrel may result in a reduction of antiplatelet activity of clopidogrel which could be life-threatening in CAD patients. Both of the interactions can be prevented by the replacement of one of the drugs. A report indicated the possibility of drug interaction in more than $90 \%$ of the prescription out of which $47 \%$ were moderate and $53 \%$ were major. Overall incidences of drug-drug interaction in the present study were quite less compared to this study. ${ }^{12}$ An average number of drugs per prescription was approximately ten; this definitely indicates polypharmacy practice. This can be dangerous as chances of drug interactions are extremely high. However, considering the severity of disease in these patients, it becomes almost impossible. One of the criteria for assessing rational drug usage is a number of drugs prescribed from the WHO essential drug list. In the present study, we observed that only $40.12 \%$ of prescribed drugs are included in the WHO essential drug list 2017. There are a good number of reports where 90$100 \%$ of prescribed drugs were included in the WHO essential drug list. ${ }^{5}$ In one scientific study WHO drug prescribing indicators were considered and adhered to ICMR guidelines in case of diabetic patients, which supports our study results for the use of WHO core indicators. $^{18}$ The best idea to prevent drug-drug interaction by choosing drugs which are less likely to produce serious drug interactions. We observed that majority of the prescribed drugs are not included in the WHO Essential drug list. In our study, stress was observed as a risk factor of CAD. There was a significant correlation between stress and coronary artery disease $(\mathrm{p} \leq 0.05)$. Previous studies showed that chronic stress predicts the occurrence of coronary heart disease (CHD). Individuals having work-related stress and who are socially isolated or lonely have an increased risk of a first CHD event. ${ }^{14,15}$

\section{CONCLUSION}

Various risk factors existed in the majority of the study population which includes high age, higher BMI, stress and pre-existence of diabetes, hypertension and other cardiovascular diseases and they have contributed to a large extent in present patient condition. The study revealed some important findings which can impact the healthcare of CAD patients. Polypharmacy was practised, which had raised a significant possibility for drug interactions. Some of these drug interactions were serious. The practice of prescribing drugs beyond the essential drug list was quite prevalent. The practice of prescribing using brand names against generic was quite frequent.

\section{ACKNOWLEDGEMENTS}

Authors are thankful to Ramanbhai Patel college of pharmacy and Dr. Jivraj Mehta smarak and health foundation for their support during the project. Authors would also like to thank all the patients for their cooperation in giving detail clinical history.

Funding: No funding sources

Conflict of interest: None declared

Ethical approval: The study was approved by the Institutional Ethics Committee

\section{REFERENCES}

1. Lopez AD, Murray CC. The global burden of disease, 1990-2020. Nat Med 1998;4:1241-3.

2. Dawalji S, Venkateshwarlu K, Thota S, Venisetty PK, Venisetty RK. Prescribing pattern in coronary artery disease: a prospective study. Int J Pharm Res Rev. 2014;3(3):24-33.

3. Tasneem S, Fouzia N. Drug utilization study in ischemic heart diseases associated with diabetes and hypertension. Int $\mathbf{J}$ Pharma and Bio Sci. 2010;1(3):1-4.

4. Maas AH, Appelman YE. Gender differences in coronary heart disease. Neth Heart J. 2010;18(12): 598-603.

5. Biradar S, Nayakawadi N, PathiIndu, Rao M, Warad V. Prescribing pattern, drug utilization and clinical pharmacy services in acute coronary syndrome patients: Am J Drug Dis Dev. 2017;7(2):63-9.

6. Sekhri T, Kanwar RS, Wilfred R, Chugh P, Chhillar M, Aggarwal R, et al. Prevalence of risk factors for coronary artery disease in an urban Indian population. BMJ open. 2014;4(12):e005346.

7. Koria B, Kumar R, Nayak A, Kedia G. Prevalence of diabetes mellitus in urban population of ahmadabad city, Gujarat. Natl J Community Med. 2013;4(3):398-401.

8. Bastenie PA, Vanhaelst L, Neve P. Coronary-artery disease in hypothyroidism: observations in preclinical myxoedema. Lancet. 1967;290(7528):1221-2.

9. Grines CL, Cox DA, Stone GW, Garcia E, Mattos LA, Giambartolomei A, et al. Coronary angioplasty with or without stent implantation for acute myocardial infarction. New Eng J Med. 1999;341 (26):1949-56.

10. Chisari A, Pistritto A, Piccolo R, La Manna A, Danzi G. The ultimaster biodegradable-polymer sirolimus-eluting stent: an updated review of clinical evidence. Int J Mol Sci. 2016;17(9):1490.

11. Ghosh A, Das AK, Pramanik S, Saha UK. Drug utilization study in patients of acute coronary syndrome on follow-up visits at a tertiary care centre In Kolkata. Asian J Pharma Life Sci. 2012;2231:4423.

12. Thomas BR, Catherin TJ, Sabu N, Lekshmi ES, Baby N, Menaka K, et al. Prescribing Pattern of Cardiovascular Drugs-A Prospective Observational Study. Indian J Practice. 2017;10(4):287.

13. Naveen A, Sravani MR, Venkat JN. Drug utilization patterns in acute coronary syndrome at a tertiary care teaching hospital: A retrospective, non 
interventional and observational study. Pharma Innov. 2017;6(9, part H):538.

14. Steptoe A, Kivimäki M. Stress and cardiovascular disease. Nat Rev Cardiol. 2012;9(6):360.

15. Dimsdale JE. Psychological stress and cardiovascular disease. J Am Coll Cardiol. 2008;51(13):1237-46.

16. Castelli WP. Epidemiology of coronary heart disease: the Framingham study. Am J Med. 1984;76(2):4-12.

17. Ranthe MF, Petersen JA, Bundgaard H, Wohlfahrt J, Melbye M, Boyd HA. A detailed family history of myocardial infarction and risk of myocardial infarction-a nationwide cohort study. PloS one. 2015; 10(5):e0125896.

18. Acharya KG, Shah KN, Solanki ND, Rana DA. Evaluation of antidiabetic prescriptions, cost and adherence to treatment guidelines: A prospective, cross-sectional study at a tertiary care teaching hospital. J Basic Clin Pharma. 2013;4:82-7.
19. Solanki N, Patel Y. Drug utilization pattern and drug interaction study of antibiotics prescribed to orthopedic patients in private hospital. Arch Pharma Pract. 2019;10(4):114-8.

20. Solanki N, Patel V, Patel R. Prescribing trends in cardiovascular conditions: a prospective crosssectional study. J Basic Clin Pharma. 2019;10:23-6.

21. Solanki ND, Patel P. Drug utilization pattern and pharmacoeconomic analysis of antihypertensive drugs prescribed in secondary care hospital in Gujarat, India. Asian J Pharm Clin Res. 2017;10(3):120-4.

Cite this article as: Solanki ND, Patel N, Desai S, Patel V. Coronary artery disease prescribing pattern and risk factor assessment in the patients undergoing angioplasty. Int J Basic Clin Pharmacol 2021;10:51722 . 